Noname manuscript No.

(will be inserted by the editor)

\title{
Can we make sense of Relational Quantum Mechanics?
}

\section{Quentin Ruyant}

November 2017

\begin{abstract}
The relational interpretation of quantum mechanics proposes to solve the measurement problem and reconcile completeness and locality of quantum mechanics by postulating relativity to the observer for events and facts, instead of an absolute "view from nowhere". The aim of this paper is to clarify this interpretation, and in particular, one of its central claims concerning the possibility for an observer to have knowledge about other observer's events. I consider three possible readings of this claim (deflationist, relationist and relativist), and develop the most promising one, relativism, to show how it fares when confronted with the traditional interpretative problems of quantum mechanics. Although it provides answers to some problems, I claim that there is currently no adapted locality criterion to evaluate whether the resulting interpretation is local or not.
\end{abstract}

Keywords Relational Quantum Mechanics · Bell's theorem · Locality . Relativism

Université Catholique de Louvain 
Carlo Rovelli has proposed an interpretation of quantum mechanics, which he has dubbed relational quantum mechanics (RQM) (Rovelli, 1996; Smerlak Rovelli, 2007). Rovelli claims that this interpretation solves the measurement problem while preserving both the locality and completeness of quantum mechanics, all this without additional structure nor many-worlds. This is a bold claim: how is this possible? By positing that the states quantum mechanics describes are relative to the observer rather than absolute. We should abandon the idea that there is a "view from nowhere", and all our worries will be put at ease.

The idea is inspired by Mermin (1998)'s view that quantum mechanics is a "theory of relations without relata", and by Everett's relative state formulation. More recently, Bene Dieks (2002) developed a perspectival modal interpretation of quantum mechanics which also assumes relative states, although they are exploited differently. Healey (2012)'s pragmatist approach also share some similarities. Rovelli's interpetation has been discussed, among others, by Dorato (2013); Bitbol (2010); Van Fraassen (2010); Dieks (2009); Brown (2009); Laudisa (2001). However it remains a minority view, and the fact that it is not clear to everyone what the interpretation says exactly might be part of the reasons why.

This paper is an attempt to make things more clear. I expose RQM's ontology and its interpretation of theoretical structures in the first section. In the second section, I examine one of its central claims: the idea that we could know when a measurement occurs. The claim is somehow ambiguous in Rovelli's writings, and I propose three possible readings of it. In the third section, I develop the most promising option, and show, in the fourth section, how it fares when faced with the traditional conundrums of quantum mechanics: the EPR argument and Bell's inequalities.

\section{Ontology and predictions}

RQM can be primarly characterised as being commited to an ontology of "observers", measurement interactions and relative events ${ }^{1}$, and by adopting an instrumentalist stance towards theoretical structures.

According to RQM, in the world, there are observers. These are not necessarily human observers, but any physical object, such as a measuring apparatus, would qualify ${ }^{2}$. During a measurement interaction with another object, an observer witnesses an event (a discrete, occurrent entity) corresponding to this object having a determinate property relative to the observer (these properties are not necessarily finely grained physical quantities: it can be a macroscopic aggregated quantity, or, for example, position with a finite degree of precision). Quantum mechanics is what allows an observer (supposedly

\footnotetext{
1 "In RQM, physical reality is taken to be formed by the individual quantum events (facts) through which interacting systems affect one another. [...] each quantum event is only relative to the system involved in the interaction."(Smerlak Rovelli, 2007, p.2)

2 "any physical system provides a potential observer" (Smerlak Rovelli, 2007, p. 2).
} 
a human observer, capable of storing information, making inferences, understanding physics, etc.) to make probabilistic predictions about future events on the basis of past and present events. But events are all relative to the observer, and different observers can have different descriptions of the same objects. This aspect is presented by Rovelli as an extension of the principle of relativity: not only lengths and durations are relative, but also the determinacy and value of properties.

As for the interpretation of theoretical structures, the idea is that the theory provides universal, objective rules that can be applied by any observer to make their predictions (this is what Van Fraassen (2010) calls a "transcendental approach"). So the way the rules work should be explained by taking the perspective of an observer. I propose to understand predictions in terms of events that could be witnessed by one of the potential future-selves of an observer.

I will follow Smerlak Rovelli's suggestion, and adopt the Heisenberg picture of quantum mechanics, wherein wave-functions do not evolve with time, but observables do. In this picture, the wave-function can be seen as encoding past and present events that the observer has witnessed ${ }^{3}$ and evolving operators can be associated with potential future-selves of the observer performing a specific measurement at a specific time. The Born rule allows the observer to derive probabilities that some events would be witnessed by a potential future-self doing a measurement corresponding to an operator. Since the predicted measurement outcomes for different observables correspond to determinate values that are relative to different future-selves, and therefore are never combined into a single description, the usual paradoxes of quantum mechanics do not arise.

If the observer actually does a measurement, then it will witness a new event and its wave-function will "collapse". But this does not mean that a physical change occurs, apart from the instantiation of an event: it merely means that the observer, having gained new information, can update its representation ${ }^{4}$. Quantum mechanics tells us how to build wave-functions from acquired information, and this is also valid for future- $O$ : what future- $O$ is doing is merely building a new wave-function from the information it has just acquired. One could express it this way: the wave-function changes because

\footnotetext{
3 "The state $\Psi$ that we associate with a system $\mathrm{S}$ is therefore, first of all, just a coding of the outcome of these previous interactions with S." (Smerlak Rovelli, 2007, p.3) Note, however, that the idea that the wave-function merely encodes past events is not strictly true: it also incorporates a predictive content, in particular when updated after a "collapse". For example, when measuring one of two entangled particles, the properties of the second one are updated too, even though it is not directly measured and corresponds to no new event for the observer. Therefore it should count as a prediction encoded in the wave-function that concerns a future observer capable of having information on the distant particle (this is explicit in Smerlak Rovelli (2007)'s treatment of EPR).

4 "What changes instantaneously at time $t_{0}$, for $A$, is not the objective state of $\beta$, but only its (subjective) relative state, that codes the information that $A$ has about $\beta$ " (Smerlak Rovelli, 2007, p. 5)
} 
$O$ has just became a new observer with a new perspective, relative to which objects have different properties.

What the observer will measure in the future and at what time it will witness an event is not part of the predictions of the observer itself. This is because the observer is not part of its own representation, and doesn't have the same information about itself that it has about other objects (as we shall see in the following, an external observer might be able to make these predictions). For this reason, predictions must be conditionalised to potential measurements, and they should not strictly count as unconditioned probabilisitc knowledge of future events, because no probability is associated to different possible measurements. What these predictions are, and what the corresponding laws and operators represent, will mostly depend on the interpretation of probabilities one adopts. Smerlak Rovelli (2007) claim that a subjective interpretation is required for RQM, i.e. that probabilities would merely correspond to degrees of credence, but Dorato (2013) suggests instead a dispositional interpretation, where events have dispositions to produce other events, and measurements are the stimulus. An alternative dispositional account, more in line with the Heisenberg picture, would be to interpret operators at any time as dispositional entities, attributed to the interaction between the observer and the object, whose stimulus and manifestations are events. I will remain uncommited on this issue.

As we can see, the theory entertains a form of relativism about events and properties, but I think its general ontological claims ("what the world is like" according to RQM) should be understood as being absolutely true in order to avoid the self-refutation problems associated with relativism, and the relativist stance should be reserved to statements about particular entities or facts in the world.

RQM is sometimes classified as a collapse interpretation, but I think it is better classified among the multiverse theories in Maudlin (1995)'s terminology (which becomes a misnomer, since RQM does not postulate multiple universes), i.e. theories that deny that experiments have determinate outcomes. This is because the collapse postulate is not introduced as a means to complete the dynamics of the theory ${ }^{5}$ : according to RQM, the wave-function and the dynamics are complete relative to the observer. The wave-function does not give determinate outcomes because these outcomes do not exist (yet) relative to the observer, they only exist relative to potential future observers (whereas in many-worlds, they exist relative to actual future observers in different branches). So the interpretation shares more affinities with Everett's relative state formulation (as Rovelli acknowledges) than with, say, GRW.

To finish this exposition, let me warn that all this narrative should be taken with a grain of salt. The notion of observation used in science, associated with sophisticated controlled measurements, is certainly different from

\footnotetext{
5 Rovelli gives, as a rationale for the collapse, that acquiring new information involves interacting with the system, which breaks the linear dynamic. But this is somehow misleading: dynamics, in the sense of laws of nature governing the phenomena, need not be involved if wave-functions are mere bookkeeping devices.
} 
the notion of subjective observation philosophers entertain, and to my knowledge, RQM does not pretend to take position in the philosophy of mind $^{6}$. Arguably, when scientists apply quantum mechanics, they do not make claims or predictions about what they observe as individuals: they make claims about robust, theory-laden scientific reports, and they talk on behalf of their measuring apparatus. They don't base their predictions solely on "past events", but rather on a sophisticated practical and technical knowledge that allows them to produce suitable controlled environments that they can associate with theoretical models. The idea that the measurement of physical quantities could be translated by systematic and non-contextual rules into first-person reports is very naive (we've now known this for almost a century) and it is unnecessary to do science (see for example Bogen Woodward, 1988). But digging into the philosophy of experimentation would take us much too far. RQM needs only assume that an experimenter doing a scientific report has indirect knowledge of a physical event relative to an apparatus in her neighbourhood, which is not so unreasonable. For the sake of the presentation, I will stick to the idealistic scheme and simply talk about observers directly observing physical quantities at specific times.

\section{Other observers}

According to this minimal interpretation, quantum mechanics is certainly a local theory. It is even trivially local: after all, all events it predicts are attached to the observer, which is at the center of its own reference frame, and distant events simply do not exist. Representation of distant objects is purely instrumental: it serves the purpose of predicting future local events. Non-locality arguments, such as Bell's, are a non-starter: even if quantum mechanics allowed observers to act instantaneously on distant objects (under standard interpretations), the theory would still be local! But the theory also looks like a solipsist version of instrumentalism. From the perspective of an observer, other observers don't really need to exist qua observers.

The (explanatory?) role of RQM's ontology would be unclear without further postulates: what is the point of introducing an ontology of observers, where all objects are on a par, if all they can do is make predictions in their own corners? Furthermore, as human beings, we never witness events directly corresponding to physical properties (at least not without a good scientific training!), so shouldn't we be able to talk meaningfully about events occuring from the perspective of our measuring apparatus? There seem to be a tension between Rovelli's naturalistic stance, which motivates the interpretation, and the radical relativism it entertains.

However, Rovelli introduces an ingredient that can help us escape this dire situation: the possibility of knowledge about external events. The idea is the following: if, during a measurement interaction described by an exernal

\footnotetext{
6 Although it would definitely be compatible with a panpsychist, or panexperientialist position.
} 
observer, the properties of two objects are correlated, then the measuring object has witnessed an event corresponding to its object having a determinate value for the measured property. It is even possible for the external observer to predict (probabilistically) the time at which the event occurs (Rovelli, 1998). These correspond to well-defined observables in quantum mechanics that the observer can measure. So, one does not have to be a solipsist. Even better: we now have a way to fill the gap of traditional collapse interpretations, since an external observer can tell when the collapse occurs. ${ }^{7}$

What Rovelli provides, here, is apparently a new interpretational rule from which an observer can interpret its measurement, so as to attribute to other objects what happens to itself (events). Of course, the rule itself is not something we could learn by experience and corresponds to no observable of the theory, so it has to be part of the universal claims of the interpretation. But its status is unclear, and three readings are possible:

Relationism: external events are real (asymmetric) relations between two objects, just as the events an observer witnesses are real relations between it and an external object.

Relativism: external events are relations between two objects, but they merely exist relative to the observer that infer them from its measurements.

Deflationism: external events merely correspond to the fact, for the observer, to have a given outcome for a specific measurement on other objects.

Relationism is not prima facie a bad option: it is rather intuitive. It is a straightforward way to escape instrumentalism. The main problem is that it reintroduces non-locality. Typically, in an EPR experiment, one can predict that a distant event will be correlated with a local event, and if these events are real, then Bell's inequalities can be formulated and, as in standard quantum mechanics, they will be violated. However it is not irrational to accept this. One could argue along these lines: quantum theory is primarily a theory about events witnessed by a single observer, and this theory is (trivially) local, but a metaphysical layer allows us to postulate external events on top of our representations, and although this layer is non-local, it does not really matter because it is metaphysical and corresponds to no predictions.

The resulting view would not come very far from the perspectival modal interpretation (PMI) (Bene Dieks, 2002) which is also commited to relative states. The main difference is that PMI postulates continuously evolving states instead of events, and relies on the bi-orthonormal decomposition to attribute a determinate relative state to any object at any instant, while RQM apparently relies on a fixed set of properties associated with types of systems to know what is measured. ${ }^{8}$ In RQM, the wave-function plays a more instrumental role, and

\footnotetext{
7 There might be a "preferred basis" problem here. What counts as a measuring interaction, and what doesn't? Are there objective criteria to tell, and are they sufficient to select a unique property that is measured? However, I will leave these issues aside.

8 According to Rovelli, bi-orthonormal decomposition is irrelevant because associated observables would generally correspond to an "uninteresting and practically non measurable quantity", while we are interested in "certain self-adjoint operators only, representing observables that we know how to measure" (Rovelli, 1996).
} 
state-relativity is addressed differently ${ }^{9}$. However, under this reading, both interpretations would be commited to relational entities between objects, and be compatible with a (structural) realist stance (see Ladyman Ross, 2007, p. 188)).

In any case, Smerlak Rovelli (2007) explicitely reject relationism: there cannot be absolute facts; everything (presumably concerning particulars) should be evaluated from a given perspective. One should not attempt to combine the views of different observers in a single picture. Although very clear on this, they remain somehow ambiguous on the right way to understand this new principle and oscillate in their writings between the two remaining options.

Let us examine deflationism first. I would say that it amounts to adopt a pragmatic evaluation of statements concerning other observers: in line with the theory of truth developed in the pragmatist tradition of Peirce and James, these statements should be evaluated in terms of their practical success for an agent, relative to the intentions of the agent. In the narrow context of physics, practical success is predictive success, and the intentions of the agent could correspond to the measurement she intents to perform. In this context, the interpretational rule given above can be seen as some kind of meaning postulate for the terms "event" and "measurement" when applied to external objects: it tells us how to evaluate, pragmatically, statements about other observers. And this is all there is to understand about these statements.

Note that a measurement need not be actually performed for the corresponding statement to be pragmatically true: it suffices that it could have been performed at the time and that the result would be obtained with certainty, because then, its truth directly follows from information the observer already has ${ }^{10}$. The view is not a pure verificationism (the deficiencies of which are well known). However, there is nothing that the statements about other observers say beyond these potential measurement results.

I don't think that deflationism is really tenable. First, it looks a bit as if we have been tricked by Rovelli's rhetoric: RQM seemed, at first sight, to offer new perspectives for interpreting quantum theory, but at the end, all we are left with is measurement results. These other observers witnessing events: that was just a way of talking. Despite all appearences, the additional rule does not really claim that the external events inferred from a representation and the events directly witnessed by an observer share a common nature. Not only does it seem to contradict the naturalistic stance endorsed by Rovelli, but this poses an epistemological problem, as noted above: since human observers

\footnotetext{
9 PMI assumes relativity to a reference, which is a system containing the described system, while RQM assumes that it is a separate system (however PMI takes the complement of the reference to be the "perspective", and this is a separate system). This means that in PMI, an object can have a state relative to itself, that the universe has a state relative to itself, and that any object has a state relative to any perspective, which is not the case in RQM.

10 This is clear in RQM when Rovelli analyses the observable corresponding to a measurement apparatus being correlated with a measured object: the expected probability is 1 , from which he concludes that the apparatus has measured the object, without requiring that the observer actually performs the measurement. This observation is analog to the principle of reality involved in the EPR argument, that will be examined below.
} 
never witness physical properties directly, and since we have no systematic rule to associate our subjective observations to them, shouldn't scientists be able to talk about events that are relative to their apparatus? And finally, the way locality problems are solved is rather trivial, as also noted above: what would count as non-locality with such an interpretation?

In the end, RQM thus understood does not seem to be distinctive as compared to, for example, QBism. QBism is the view that quantum mechanics is not a theory about the world, but about our degrees of credence concerning predictions. The theory provides universal, objective rules for updating these degrees from the information one gets on the world through events. All this is shared by RQM. One difference is that QBism is human-centered, while RQM is not: any physical object qualifies as a potential observer. But what remains of it if all talk of external observers boils down to talk of events relative to us? If anything, RQM is more radically instrumentalist than QBism: after all, the latter assumes that events are objective and publicly accessible...

For these reasons, I think we should take seriously the idea that observers really know about external events. The interpretative rule Rovelli provides really tells us how to interpret our measurements, and if observers each can assume this rule, then they should really talk about other observers, not merely about their future observations. I think this is essential to do justice to the naturalistic stance (according to which all objects are on a par) that Rovelli mentions to motivate his interpretation. Adopting this reading also allows one to defuse some of the intuitive arguments against RQM. For example, Laudisa (2017) cites Wigner, who argued that it would be absurd to think that a friend that we represent in a superposition of state within an experimental situation has not witnessed a determinate outcome. But with this reading, this is not exactly what RQM says.

So if neither relationism nor deflationism is the right way to interpret RQM, only one option remains: relativism. In this option, it is true, from the perspective of a given observer, that other observers witness events, and these events are indeed of the same nature as the ones the observer directly witness. This makes the interpretation distinct from an instrumentalism. However, all this is true relative to an observer, and this could help RQM keep its promises: being at the same time complete and local, without the many-worlds.

In what follows, I will assume that this is the right way of understanding RQM and examine how far one can go with it.

\section{Relativism}

The idea of the relativist option can be expressed as follows: basic facts about events witnessed by an observer allows it to deduce more extended facts about the world; these facts are not equivalent to the basic facts from which they are deduced: they say more than the basic facts (thanks to the interpretational rules given by RQM); however, these facts are not absolutely true, but only true relative to the observer, as are the basic facts form which they were 
deduced. This is the characteristic captured by the slogan: "there is no view from nowhere". So in a sense, we have a double relativity when it comes to events witnessed by other observers: these events are relative to the other observer, relative to the first observer.

This relativity extends to predictions in the following way: if quantum theory allows an observer $O$ to predict future events, then the basic facts concerning these events are relative to a potential future-self of $O$, and the prediction also concerns the extended facts that could be deduced from these basic facts, but these are also relative to the potential future-self of $O$. And the predictions are relative to $O$-now.

Relativism differs from deflationism in the following way: arguments regarding locality or completeness need not be applied to basic facts only; they can also be applied to extended facts that concern distant objects, taken at face value, and, possibly, there would be a well-defined sense of "locality" in this interpretation, and quantum theory would pass the test.

This option can be captured by adopting a relativist conception of truth, where truth is a two place predicate indexed to an observer. This also applies to second-order statements, such as "p is true-for- $O$ ": this statement only has a truth-value relative to an observer (be it $O$ or another). Let us assume the following principles:

Basic facts principle: If an observer $O$ can predict with certainty the outcome of a measurement that it could perform at $t$, then there is a corresponding basic fact (given the interpretational rules of standard quantum mechanics) for $O$ at $t$ (even if $O$ did not actually perform the measurement).

Extended facts principle: If an extended fact can be deduced (with the interpretational rules of RQM) from basic facts that are true for $O$ at $t$, then it is true for $O$ at $t$.

Disquotational truth principle: from the perspective of $O$, " $p$ is true-for- $O$ " is equivalent to $p$.

But now here is a puzzle: if, taking the perspective of $O$, it is true-for- $P$ that $S$ has a determinate spin-x, and assuming that possible values for spin-x are -1 and +1 , then, as a matter of pure logic, it is either true-for- $P$ that $S$ 's spin-x is -1 , or it is true-for- $P$ that $S$ 's spin-x is +1 . Indeed, we do not mean that $P$ has witnessed a disjunction of values for the property, but that $P$ has witnessed one of the two. But none of the statements in the disjunction correspond to basic facts for $O$, so if quantum theory is complete, they must both be false-for$O$, and their disjunction is false as well! Assuming an ignorance interpretation of probabilities for our two statements would ruin the whole story, since all the paradoxes of quantum mechanics would show their ugly face again. But then, we cannot claim that $P$ has seen a determinate property for $S$, in pain of contradiction.

So how can a disjunction be true-for- $O$ while all of its terms are false-for$O$ ? Perhaps its terms could be neither true- nor false-for- $O$, rather than false. It seems that the relativist must drop the bivalence of truth to accomodate 
its position ${ }^{11}$. This, somehow, makes sense: after all, relative truth means something like "knowledge" (or "having information on") in RQM, and there is no contradiction in knowing that a football team has either won or lost the match without knowing the final result. RQM only takes a more robust approach towards this, by forbidding an ignorance interpretation. One would not want to revise classical logic too much to accomodate this, but formally, this can be done with modal operators (so for example, we can have $(p \vee q)$ and $\neg p$ and $\neg q$ without contradiction, and in this context, the disquotational property of truth is a theorem of modal logic: $p \rightarrow p$ ). I won't explore this any further, but it's important to note that the way to make sense of this option is not trivial, and other difficulties that were not mentioned here might arise. One should be careful when establishing the rules that allow an observer to infer extended facts.

In any case, this relativist aspect (which is also present in the deflationist view, although it doesn't encounter the difficulty just mentioned) has sprung lots of discussions, in particular in Van Fraassen (2010) and Brown (2009). This is the most counter-intuitive part. Couldn't it be true-for- $O$ that "it is true-for- $P$ that $p$ ", but false-for- $P$ that $p$ ? There seem to be no contradiction, but it would be as if $O$ and $P$ lived in different worlds, without any constraint between these worlds. Certainly, if the theory permits that, it is absurd (or at least not very far from a many-world interpretation). But according to RQM, these statements have no meaning if not evaluated from an observer's perspective, and if we take, for example, $O$ 's perspective, they become contradictory (assuming the disquotational principle), so our absurd situation is excluded. The rules of quantum mechanics also ensure that it cannot arise from a third observer's perspective either. One could legitimately remain unsatisfied, and crave for absolute facts. But Rovelli urges us, citing Wittgenstein, to take a quietist approach towards this.

Yet without any "transcendental" constraints on what different observers see, it is not clear in what sense they can be said to describe the "same" situation differently, as explained by the main narrative of Rovelli (1996)'s presentation: what is "the same"? Van Fraassen (2010) proposed some additional principles to tame this disconfort. In our context, this would amount to requiring that if something is true-for- $O$, then it cannot be false-for- $O^{\prime}$, where $O^{\prime}$ is any system containing $O$ (van Fraassen expresses this in terms of orthogonality of relative states): this ensures a transcendental coherence between what different observers observe. The problem is that these statements are already true in quantum mechanics, from the perspective of any observer and given the relativist interpretational rules of $\mathrm{RQM}^{12}$. In any case, the whole picture is still relativist.

I won't dwell on whether one can make sense of the couterintuitive, radical relativism of RQM, and accept it for the sake of argumentation. In the follow-

\footnotetext{
11 Ladyman Ross (2007, p. 188) claims that RQM requires intuitionist logic.

12 Perhaps they could allow inferences concerning what is possibly true for an external observer, in a way that is not already allowed by the rules given by Rovelli. This remains to be examined.
} 
ing, I rather wish to focus on an easier question: is RQM, thus formulated, a complete and local theory? In order to answer this question, I will examine in turn the EPR argument and Bell's inequalities.

\section{The locality and completeness of RQM}

The EPR argument is an argument to the effect that quantum mechanics is necessarily an incomplete theory. It rests on a few postulates: separability, locality, and the principle of reality, which is a criterion for assessing that there are "elements of reality": if an observer knows, without interacting with a system, that a measurement would yield a given result with certainty, then there is an element of reality corresponding to it. This is very close to our principle for basic facts above.

An informal version of the argument (in a classical context) runs as follows:

1. Assume an entangled pair of particles $a$ and $b$, such that the sum of their spin in either direction must be 0 at the end of an experiment. Assume two observers $O$ and $P$ separated by a space-like interval that can measure the spin of $a$ and $b$ in any direction. $O$ measures the spin-x of $a$ and obtain a determinate result.

2. In this situation, the result of a measurement of the spin-x of $b$ by $P$ would give an opposite result with certainty.

3. If this is so, then according to the principle of reality, there is an element of reality corresponding to $b$ having a determinate value for spin-x.

4. Given locality and separability, this element of reality is not caused by $O$ 's measurement, and it would have existed even if $O$ hadn't performed the measurement.

5. $O$ could have measured spin-y, and the same reasoning entails that there is an element of reality corresponding to $b$ having a determinate spin-y. Given separability, this element of reality must exist even if $O \operatorname{did}$ not perform this measurement.

6. Then there are elements of reality corresponding to $b$ having a determinate spin-x, and to $b$ having a determinate spin-y.

7. But quantum mechanics precludes a particle having both a determinate spin-x and spin-y. Therefore, quantum mechanics is incomplete.

The premise of the argument that RQM denies is, of course, the criterion for assessing that there are elements of reality (contra Laudisa, 2017): despite their similarities, it is different from our principle about basic facts, because for RQM, facts are relative to the observer rather than absolutely true.

Now here is how the relativistic stance of RQM allows one to defuse the argument: taking the perspective of $O$, and even assuming that $b$ has a determinate spin-x for $O$ measuring spin- $x$ and that $b$ has a determinate spin-y for $O$ measuring spin-y, these are different potential observers, and they cannot both exist. So it is not true, for any actual observer, that $b$ has both a determinate spin- $x$ and a determinate spin-y, and there is no reason to think that 
quantum mechanics is incomplete. It can well be complete, in the sense that it would describe all properties objects have relative to a given perspective.

As we can see, RQM indeed provides an answer to the EPR argument. A further observation, that will be important for the following, is that these facts associated with $b$ are not even true for $O$ at the time it performs a measurement on $a$. Following are principle of extended facts, they are only true for $O$ at a later time, when it can actually perform a measurement that would allow it to acquire information on $b$.

This leads us to Bell's inequalities. Bell has introduced his theorem as an elaboration of the EPR thought experiment exposed above, but the theorem is more focused on locality than on completeness: Bell introduces inequalities that a theory must respect in order to be a local theory, and shows that quantum mechanics violates these inequalities. Experimental tests of the inequalities have been performed since then (Aspect et al., 1982), which seems to imply that any empirically adequate theory must be non-local. But is there an unquestioned realist premise in Bell's argumentation?

I will concentrate on what has came to be called "outcome independence". This criterion for the predictions of a theory can be expressed as follows:

$$
\begin{aligned}
& P_{w}(\beta \mid A, B, a)=P_{w}(\beta \mid A, B) \\
& P_{w}(\alpha \mid A, B, b)=P_{w}(\alpha \mid A, B)
\end{aligned}
$$

Here, $w$ is the wave-function from which predictions are made: the state of the world according to the theory. $A$ and $B$ correspond to properties measured on the system at a space-like interval, and $\alpha$ and $\beta$ correspond to the outcomes of these measurements. In substance, outcome independence says that the probability that an outcome is obtained should not depend on a distant outcome, but only on the state of the system from which the predictions are derived (this means that there can be distant correlations only if they are already encoded in the initial state).

Let us consider an observer $O$ measuring $A$ on one side of the setup, and an external observer $P$ performing measurement $B$ on the other side at $t$. I will assume that $\alpha$ and $\beta$ can take value +1 or -1 with half probability, and that the particles are entangled in such a way that these values must be opposite at the end of the experiment. Then outcome independence is violated in the following way. Take the statements:

$-p_{a}$ : there is an event (measured by $O$ at $t$ ) with outcome +1

$-p_{b}$ : there is an event (measured by $P$ at $t$ ) with outcome -1

According to quantum theory, we have $P\left(p_{b} \mid p_{a}\right)=1$ and $P\left(p_{b}\right)=0.5$, which, if $p_{a}$ and $p_{b}$ are separated by a space-like interval, violates outcome independence.

In the context of RQM, these inequalities should be evaluated from the perspective of an observer situated at the beginning of the experiment, that makes predictions about facts and events for its future-selves. I will call $O_{1}$ the future-self of $O$ that measures the system at $t$ and $O_{2}$ the future-self of 
$O$ that gathers information on $P^{\prime}$ 's measurement at $t^{\prime}>t$. This requires that $P$ at $t$ and $\mathrm{O}_{2}$ at $t^{\prime}$ be separated by a time-like interval. The two statements become:

$-p_{a}^{\prime}$ : there is an event relative to $O_{1}$ with outcome +1

- $p_{b}^{\prime}$ : there is an event relative to $P$ at $t$ with outcome -1 , relative to $\mathrm{O}_{2}$.

Following relativism, statement $p_{b}^{\prime}$ must be relative to $\mathrm{O}_{2}$ because it corresponds to extended facts that can only be deduced from information available to $\mathrm{O}_{2}$.

The problem for transposing Bell's inequalities to RQM is that the facts that are predicted are no more absolute facts located at a point in space-time. They can be located at a point in space-time, but they are also indexed to an observer, which is located elsewhere. Typically, $p_{b}^{\prime}$ concerns $P$ at $t$, but it is relative to $\mathrm{O}_{2}$ at $t^{\prime}$. And this is how Smerlak Rovelli (2007) pretend to solve the problem of locality: since information on $P$ must travel back to $\mathrm{O}_{2}$, and since no information travels faster than light, locality is not violated.

This is rather intuitive, but itthe argument deserves a more precise analysis. How shall we evaluate locality in RQM? What would count as a non-local interaction in this picture? When are relative facts separated by a space-like interval? There are three options:

1. Locate relative facts according to the event they describe, and keep Bell's criterion

2. Locate relative facts according to the observers they are relative to, and keep Bell's criterion

3. Take relative facts as asymmetric relations between two objects, and comeup with a locality criterion taking into account both relata.

In the first option, RQM is non-local, because predictions concerning distant facts are statistically dependent, as in standard quantum mechanics, and the relativity of facts is simply irrelevant. This solution (which would be what we would have obtained in a relationist picture) seems unfit, because it does not take into account the fact that facts are relative.

In the second option (which is what we would get in the deflationist picture), RQM is trivially local, because distances are simply irrelevant: only the observer is relevant. This is no more tenable than the first option for the reasons given before: there is no way any theory could be non-local in this picture, even if people could act on distant objects at their will. Note that the fact that extended facts are inferred from basic facts is not a sufficient reason to choose this option: under a non-deflationist view, extended facts are not equivalent to basic facts, and there is no reason not to take into account their position in space-time.

So our locality criterion should involve both the spatio-temporal position of the fact and that of the observer to which it is relative.

One could consider the following criterion: any fact relative to an observer should be situated in its past light-cone. This means that an observer can have no direct knowledge about distant events. In the case of EPR, $P$ 's measurement result is not a fact for $O_{1}$ : it can only be a fact when $O_{2}$, because $P$ 
is in the past-light cone of $\mathrm{O}_{2}$. This seems reasonable, and it does justice to Smerlak Rovelli's treatement of EPR, where they emphasise the importance of interacting for acquiring information. However, no statistical independence between different facts is really involved in this criterion, and this condition can be achieved only by specifying the interpretative rules from which extended facts are deduced from basic facts. Non-locality is stipulated rather than specific to a given theoretical structure. One could violate this criterion by allowing an observer to deduce distant extented facts from local facts (for example, by allowing $O_{1}$ to deduce $P$ 's measurement). So arguably, any theory still could qualify as local if suitably interpreted (even if communication at a distance is feasible, so long as the result can only be witnessed by a future observer). The criterion seems insufficient.

One could observe that standard quantum mechanics, without the collapse postulate, is a local theory. The difficulty here is that neither the wave-function nor its dynamics have an ontological interpretation in RQM, so it is not clear how this locality translates in this interpretation.

One could consider a criterion that mimics parameter independence, where any outcome must be statistically independent from what the measurements that are carried out at a distance (not their outcomes), along these lines: a fact about a distant event should not depend on the measurements the observer has performed so far, unless this was in the past light-cone of the event. Then quantum theory would pass the test, but this has nothing to do with RQM since quantum theory does not violate parameter independence, and RQM does not solve any problem specifically.

Finally, one could recall the main motivation of locality: compatibility with the theory of relativity, and build a criterion from relativity considerations. This is perhaps the most sensible approach. But without a truth-relativist interpretation of relativity theory, it is hard to tell what the resulting criterion would look like.

There are perhaps other possibilities. In any case, Rovelli's claim that RQM saves the locality and completeness of quantum mechanics is hard to assess if no truth-relativist criterion for locality is proposed. It provides a solution to the EPR argument, but the way it would address Bell's inequalities is unclear. For this reason, I do not think that the issue is really settled.

\section{Can we make RQM more realist?}

The radical relativism of RQM, as understood here, brings some incomfort. We could intuitively express the worry this way: the external observers that an observer $O$ knows about are not the real observers, they are mere observers for $O$, and there is no guarantee that the real observer's observations match $O$ 's predictions, even though the general (absolute) statements of the interpretation assumes that there are observers and events.

Van Fraassen (2010) proposed some additional principles to tame this disconfort. In our context, this would amount to requiring that if something is 
true-for- $O$, then it cannot be false-for- $O^{\prime}$, where $O^{\prime}$ is any system containing $O$ (van Fraassen expresses this in terms of orhogonality of relative states): this ensures a transcendental coherence between what different observers observe. The problem is that these statements are already true in quantum mechanics, from the perspective of any observer and given the relativist interpretational rules of RQM, but perhaps they could allow inferences concerning what is possibly true for an external observer. In any case, the whole picture is still relativist.

In this section, I wish to briefly explore another possible way to alleviate this worry: assuming presentism.

Indeed, the relativity of truth that characterises RQM, as interpreted here, is mainly employed to eliminate non-locality when it comes to considering predictions about future events, but it is unnecessary with regards to events in an observer's past light-cone, so one could well consider that these events are absolutely real, or, at least, that it is absolutely true that they are real-for- $O$, and eliminate the double-relativity of statements about others (on the ground that other observers' events are also absolutely real-for-them). This move does not seem to affect the way RQM can answer the puzzles of quantum mechanics if the principle is denied to potential future observers (because they do not yet exist), and it has the capacity to put at rest most of the worries associated with RQM: after all, we all live in the same world, because we all share a common past. ${ }^{13}$

A problem with presentism is that it seems to require a preferred frame of reference so as to have a well-defined notion of simultaneity, which is problematic with regards to relativity theory. However, another interpretative rule could solve this issue by appealing to decoherence.

In this section, I wish to explore another possible extension of RQM, based on decoherence.

First, let us recall the rationale for assuming that distant events should not count as facts for an observer. Take the case of the EPR experiment above: why does $P$ 's measurement counts as a prediction for $O_{1}$ rather than a fact? This is because $O_{1}$ cannot directly measure $P$. In principle, someone could intervene in the experiment and make a quantum measurement on $P$ and the measured particle that would (in colloquial terms) display interferences between the two possible results for the measurement. This would have as an effect that there would be no answer to the question "what did $P$ see?", just as in a double-slit experiment, there is no answer to the question "in which slit did the electron pass?".

However, there are situations where such a scenario is implausible. Decoherence is a phenomenon by which a system that interacts with an environment becomes indistinguishable from a statistical mixture for a certain preferred basis (Zurek, 2003). Quantum correlations in the system don't really disappear: rather they are, loosely speaking, diluted in the environment. Concretely,

\footnotetext{
13 There might also be interesting connections between relativist truth and the use of a tensed semantic associated with presentism. See also Dorato (2013) for considerations on time and becoming in the context of RQM.
} 
this means that measurements of the kind describes above become practically impossible, because they would involve a finely tuned control of the environment. Now, (notwithstanding potential locality problems) that could be seen as a reason to assume that " $P$ has measured the particle" $s$ spin- $x$ " is a fact for $O_{1}$, because nothing could prevent any potential future- $O_{1}$ from incorporating this as a fact. In sum, one could add the following principle to the ones mentioned above:

Distant fact principle: if $O$ can predict that a fact is true for all its potential future-selves at a given time, then it is a fact for $O$ now.

Our new interpretative rule means that an observer can infer extended facts beyond its past light-cone. This also includes many facts situated in its future light-cone: roughly all facts that can be predicted classically are concerned (the position of planets for centuries), which means most macroscopic ones, so long as they are not affected by unsettled micro-events. A problem with this proposal is that it might reintroduces non-locality, depending on what criteria one adopts. However, decoherence has as an effect to make the violations of Bell's inequalities less plausible (Levkovich-Maslyuk, 2009). The issue should be examined in more details.

Combined with the presentism suggested here, our new interpretative rule means that we have a (probabilistic) means to determine the existence of absolute events outside of our past light-cone, i.e. an observer can know what events are "present" or not, without having to assume a preferred reference frame.

A problem with this proposal is that it probably reintroduces non-locality. Decoherence can have as an effect to make the violations of Bell's inequalities implausible. However, at the beginning of an EPR, one could predict from our new principle that $P$ 's measurement will be a fact for $O_{1}$, and depending on our locality criteria, this could be a mark of non-locality. Another issue is that we could have to accept that many events in our future light-cone are actually "present" (or at least that they are absolute facts about the future): roughly all facts that can be predicted classically are concerned (the position of planets for centuries), which means most macroscopic ones, so long as they are not affected by unsettled micro-events. This also means that coarse-grained events associated with wholes can be "present" while events in their parts at the same time are not, and that micro-facts in our past are not yet "present" if they have not decohered (all this is very counterintuitive, but not necessarily absurd if "present" just means "settled fact"). So rather than a growing-block theory of time, we have something like a solidifying-block theory, where solidification (the process of events) is not uniform and is a scale-sensitive phenomenon. All this looks a bit crazy, but in the end, these are only useful representations for predicting occuring events... The main problem is that we lose a connection between this notion of present and the notion of simultaneity in relativity theory or in classical physics.

And of course, all this is hardly a fully-developed proposal at this stage. 


\section{Conclusion}

RQM is an inspiring interpretation of quantum mechanics, but an ambiguity remains in the way it addresses external events. In this paper, I attempted to make things more clear by considering three distinct options. Only one, radical relativism about facts, can make the position distinct from an instrumentalism while remaining faithful to the way the interpretation is formulated by Rovelli. It can answer the EPR argument against completeness. However, whether (and in which sense) this option provides a local theory is unclear as it stands.

In any case, I think the interpretation deserves more consideration than it has received so far in the literature, and I hope this paper has contributed to make it more clear.

\section{References}

Aspect, Alain, Dalibard, Jean Roger, Gérard (1982). Experimental Test of Bell's Inequalities Using Time- Varying Analyzers. Physical Review Letters, 49(25), 1804-1807.

Bene, Gyula Dieks, Dennis (2002). A Perspectival Version of the Modal Interpretation of Quantum Mechanics and the Origin of Macroscopic Behavior. Foundations of Physics, 32(5), 645-671.

Bitbol, Michel (2010). De l'intérieur du monde. Champs-Flammarion, Paris.

Bogen, James Woodward, James (1988). Saving the phenomena. Philosophical Review, 97(3), 303-352.

Brown, Matthew J. (2009). Relational quantum mechanics and the determinacy problem. British Journal for the Philosophy of Science, 60(4), 679-695.

Dieks, Dennis (2009). Objectivity in Perspective: Relationism in the Interpretation of Quantum Mechanics. Foundations of Physics, 39(7), 760-775.

Dorato, Mauro (2013). Rovelli's relational quantum mechanics, anti-monism and quantum becoming.

Healey, Richard (2012). Quantum Theory: A Pragmatist Approach. British Journal for the Philosophy of Science, 63(4), 729-771.

Ladyman, James Ross, Don (2007). Every Thing Must Go: Metaphysics Naturalized. Oxford University Press.

Laudisa, Federico (2001). The EPR Argument in a Relational Interpretation of Quantum Mechanics. Foundations of Physics Letters, 14(2), 119-132.

Laudisa, Federico (2017). Open Problems in Relational Quantum Mechanics.

Levkovich-Maslyuk, F. L. (2009). Two destructive effects of decoherence on Bell inequality violation. Physical Review A, 79(5).

Maudlin, Tim (1995). Three Measurement Problems. Topoi, 14(1), 7-15.

Mermin, N.D. (1998). What is quantum physics trying to tell us? American Journal of Physics, 66, 753-767.

Rovelli, Carlo (1996). Relational Quantum Mechanics. International Journal of Theoretical Physics, 35, 1637-1678. 
Rovelli, Carlo (1998). 'Incerto tempore, incertisque loci': Can we compute the exact time at which a quantum measurement happens? Foundations of Physics, 28, 1031-1043.

Smerlak, Matteo Rovelli, Carlo (2007). Relational EPR. Foundations of Physics, 37(3), 427-445.

Van Fraassen, Bas C. (2010). Rovelli's World. Foundations of Physics, 40(4), 390-417.

Zurek, W.H. (2003). Decoherence, einselection, and the quantum origins of classical. Reviews of Modern Physics, 75, 715-775. 